\title{
A Smart Phone/Tablet based Mobile Health Care System for Developing Countries
}

\author{
Avinash S. Vaidya, M.B. Srinivas \\ BITS - Pilani, Hyderabad Campus, \\ Hyderabad, India \\ avinash.s.vaidya@gmail.com, \\ mbs@hyderabad.bits-pilani.ac.in
}

\author{
P.Himabindu \\ Wipro Technologies, Bangalore, India
}

Daria Jumaxanova

Dept. of Information Systems

Semey State University

Kazakhstan

\begin{abstract}
Increasing cost of health care in developing countries is placing heavy financial burden on its populations. With the advent of mobile and tablet technologies however, it is possible to reduce this burden to some extent through telehealthcare. In this paper, authors describe their effort to design portable diagnostic devices that can communicate to smart phones and tablets there by making tele-healthcare possible. A possible architecture of their model is presented and components thereof discussed.
\end{abstract}

\section{INTRODUCTION}

A survey [1] reports that, in $95 \%$ of countries, inflation in healthcare cost has exceeded the rate of general inflation. The increase in inflation rate has disturbed the medical facilities in rural or remote areas. Table I shows the trends in medical inflation rate. Population in remote areas and the persons below poverty are the main victims of this inflation. This has given rise for the necessity of cost effective and scalable solution for the healthcare system.

TABLE I. Global Average Medical Trends (2006-2011) [1]

\begin{tabular}{|l|l|l|l|l|}
\hline \multirow{2}{*}{ Measure } & \multicolumn{4}{|c|}{ Medical Inflation Rate/Net Inflation Rate (in \%) } \\
\cline { 2 - 5 } & $\mathbf{2 0 0 6}$ & $\mathbf{2 0 0 9}$ & $\mathbf{2 0 1 0}$ & $\mathbf{2 0 1 1}$ \\
\hline Asia Pacific & $12.2 / 8.8$ & $9.9 / 8.1$ & $10.3 / 6.6$ & $10.2 / 7.1$ \\
\hline Europe & $5.4 / 3.6$ & $9.4 / 8.2$ & $7.8 / 5.8$ & $9.1 / 7.2$ \\
\hline $\begin{array}{l}\text { Latin } \\
\text { America }\end{array}$ & $14.6 / 8.4$ & $11.6 / 4.0$ & $12.1 / 4.7$ & $13.7 / 6.1$ \\
\hline $\begin{array}{l}\text { Middle } \\
\text { East/Africa }\end{array}$ & $11.4 / 6.3$ & $10.9 / 3.7$ & $10.1 / 3.5$ & $10.3 / 4.4$ \\
\hline $\begin{array}{l}\text { North } \\
\text { America }\end{array}$ & $10.3 / 7.7$ & $10.8 / 10.8$ & $11.3 / 9.7$ & $11.6 / 10.1$ \\
\hline
\end{tabular}

The advent of technology has made portable technologies usage in healthcare systems possible. Portability feature gives the consumer an opportunity to use the device wherever required and it also plays a very important role in the field of healthcare as there are limited healthcare facilities available in rural/remote areas and it is difficult to deploy the medical facilities considering the current trends of the medical inflation. The purpose of this work is to design a portable diagnostic and communication device, so that, a unified application can be presented.
The paper will discuss related work to this area in the next section. The architecture of the system will be explained in detail in section III of the paper and implementation details of the system, which includes both hardware and software aspects will be elaborated in section IV. The system is tested in a rural area in coordination with MediCiti group of hospitals. The experimental setup and results of the test is explained in section V.

\section{RELATED WORK}

This section of the paper will describe the related studies in different aspects of the work.

There are evidences of efforts put to solve the issue of rising medical expenses. Various companies are working on providing a cost effective healthcare and some of them are using the idea of mobility to increase the user experience in the field of healthcare. Doctors are provided with a solution by which they could monitor their patients remotely [2-3]. Also there are systems which provide short message service based healthcare solutions medical records for the users [4]. But very little effort has been put towards making it suitable for low cost healthcare solutions. The main focus of most of these works is towards maintaining the patients' diagnostic data, making it available for remote viewing and reminder services.

There are commercially available devices like[5-6] which stores the data onto a computer and transmit it. Those devices mainly interact with the proprietary software, also the devices are expensive to use it as a cost effective solution for the problem in hand. It is hard to extract the information collected from the devices and process the data. All these studies point to the need of an integrated solution of hardware and software, a solution which would bring the healthcare to remote places of a developing countries in a cost effective way.

Various papers have been published on calculation of blood pressure, heart rate and electrocardiogram (ECG). Measurement of blood pressure, heart rate or ECG is no more a difficult task. There are several methods [7] used for blood pressure measurement for e.g. pulse oximetric, auscultation, pulse transit time (PTT) [8-10] etc. The methods used for the blood pressure calculation could be invasive or non-invasive. The calculation of blood pressure using pulse transit time has proven to be accurate enough and has an added advantage of continuous measurement. 
The next section of the paper will discuss about the architecture of the developed system.

\section{ARCHITECTURE}

The architecture as a whole can be viewed as a telemedicine solution. The architecture of the system focuses on the needs of healthcare in remote areas and it is built around the technology that is available at a rural area. The architecture provides a low cost and reliable solution for rural healthcare system using telemedicine.

As shown in figure 1, the health kiosk at a rural area, cloud storage and a hospital are the main components of the architecture of the developed system. The health kiosk encompasses a diagnostic device and a tablet/smartphone. The diagnostic device would communicate to the tablet/smartphone through a wired connection. A technician who is trained to operate the device collects the diagnostic data of the patient using the tablet/smartphone and stores the necessary information on a cloud storage space. The cloud space acts as the central storage space of the system from which a doctor can retrieve the data to diagnose the patient.

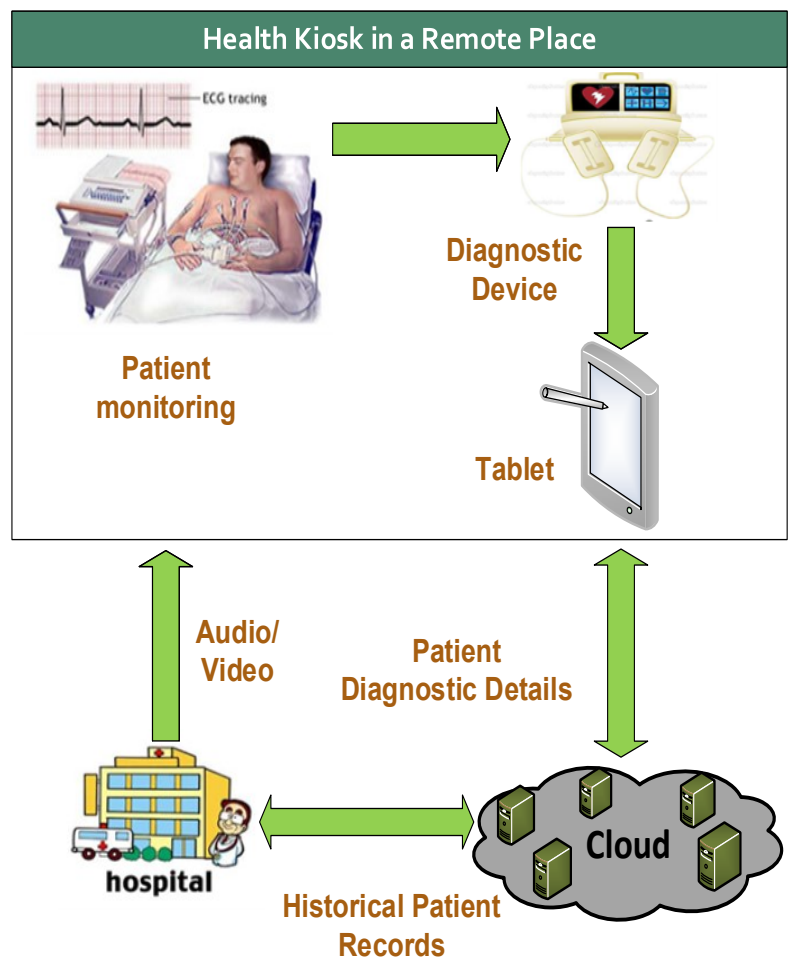

Figure 1. Architecture of the system

Doctor will communicate his/her observations and prescriptions to the remote place through the same cloud. The audio/video call feature present in the system will help the doctor to get a real time assessment of the patients status in case of physical injuries, infections etc. This feature will also enable the patient to seek a one to one counselling with the doctor.

As a whole the architecture is designed to empower the healthcare system with the technology in a cost effective method. Architecture delivers a low cost solution without compromising on the reliability of the data and safety of the patients. The next section of the paper will discuss the implementation aspect of each component of the system.

\section{IMPLEMENTATION}

\section{A. Hardware}

The developed system measures the electrocardiogram (ECG), blood pressure and heart rate of the patients. In the following implementation we have used 3-lead system to record the electrocardiogram of a human body. As mentioned in the section of related studies, Pulse Transit Time (PTT) is used to measure the blood pressure of the human body. Heart rate is measured using the Photoplethysmography (PPG) waveform, which is a non-invasive method of measuring the blood volume through optical pulses. Cypress Programmable System-on-Chip 3 (PSoC3) is chosen as the processing platform as it has the capability of mixed signal processing and also adds the compactness and portability to the system.

The magnitude of the electrical signals acquired form the ECG electrodes attached to the patient's body will be in order of millivolts. This signal is amplified using an instrumentation amplifier and the amplified analog signal is passed through a passive high pass filter and further processed using PSoC3. The schematic of the PSoC3 implementation is shown in figure 2. The analog signals acquired from the instrumentation amplifier are buffered using an operational amplifier and the buffered signal is fed to the analog to digital converter (ADC) block of PSoC3. ADC is configured to sample at three hundred samples per second and a data width of 19 bits is used. Sampling rate of ADC would further reduce the high frequency noise.

The PPG signal is acquired using a finger clip sensor. The acquired analog signal is filtered using a passive low pass filter to remove the high frequency noises. The filtered analog PPG signal is fed into PSoC3 and the input signal is buffered through the operational amplifier and digitized using the ADC. Buffered signal is sampled at a rate of hundred samples per second and a data width of 16 bit is used. The digitized samples of ECG and PPG are used to calculate the SBP and DBP [11].

PPG is also used to calculate the heart rate of the patient. The analog PPG signals are fed to a comparator as an input. The output of the comparator drives the interrupt of the Timer block of PSoC3. Time period between the pulses are calculated using the timer. Using this time period hear rate of the patient is calculated.

The samples of ECG, calculated values of blood pressure and heart rate are transmitted to a tablet. The tablet and the PSoC3 communicated through Universal Serial Bus (USB) [10]. The USB of PSoC3 is programmed to operate in a vendor specific mode. Bulk transfer protocol of USB is used in the communication.

\section{B. Software}

An android based tablet is used in the developed system. An application is developed to interface tablet with PSoC3. The application is also used to collect patients' personal information and diagnostic information. The collected data are stored in the cloud storage using mobile connection. 


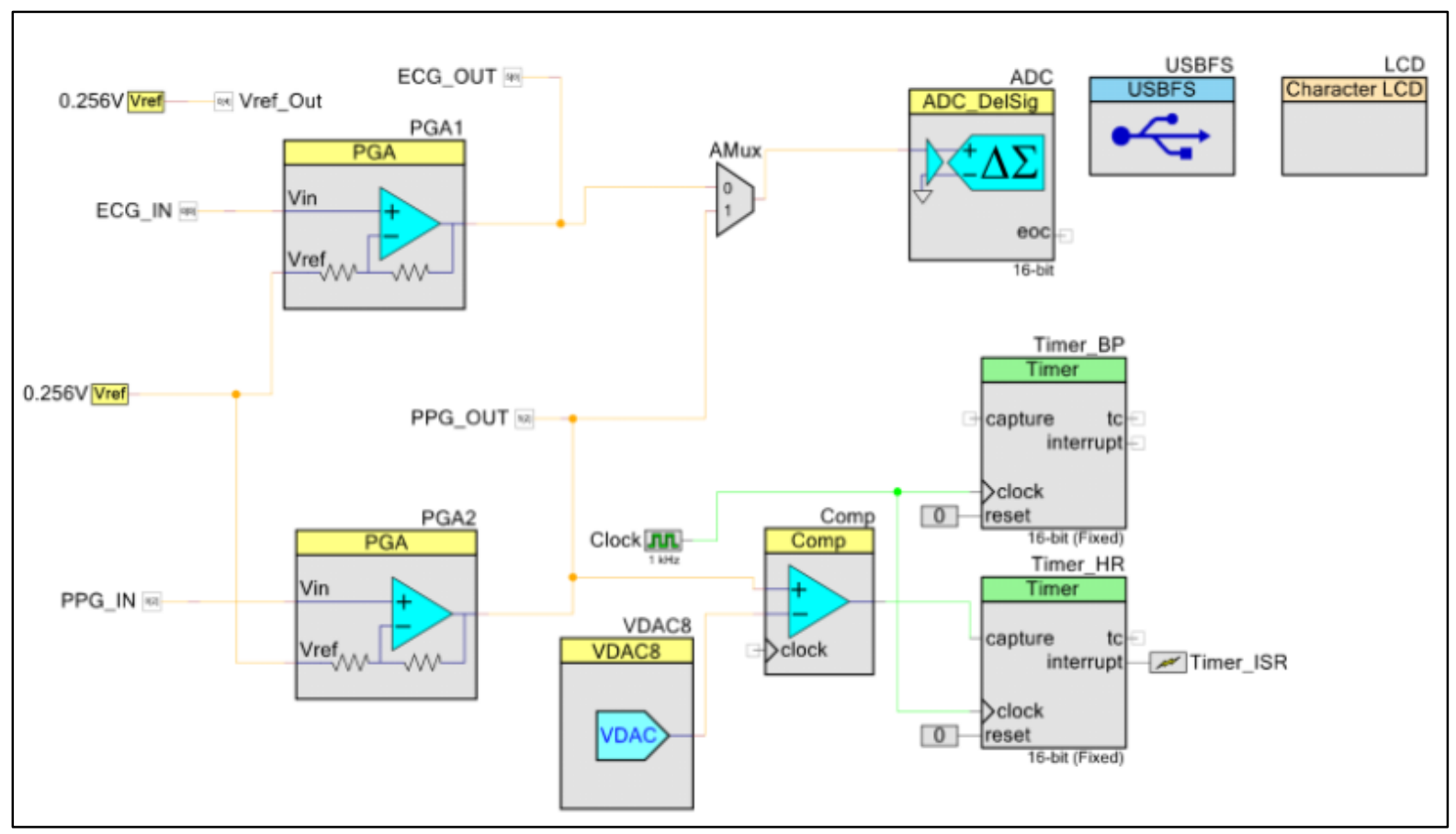

Figure 2. Schematic of PSoC3

Figure 3 shows the flow chart of the software developed. At start the trained technician enters the registered patient ID if it exist else the new patient is registered by entering the details like ID no., name, age, gender and address and the photo of the patient is also captured and stored. Once a valid ID is entered the application displays the details of patient and this window of the application also provides options to collect diagnostic data, view historical records and make an audio/video call to the doctor. The diagnostic data is collected by clicking on the buttons meant for collecting each parameter. The digitized ECG samples from the PSoC3 is reconstructed to form a graphical representation and it is stored as JPEG image. The BP and heart rate values are stored as string values. The collected diagnostic data is saved on to the cloud storage. The historical diagnostic records of the patient are displayed in a chronologically ordered list when view historical records option is selected. An option to view the cumulative graph of $\mathrm{BP}$ and heart rate reading is also provided. The audio/video call option establishes an audio/video call [12] with the distant doctor and the patient. A replica of the software is used at the doctor's end to enable the doctor to view patients' records and give his/her observations and prescription. Figure 4 shows the screenshots of few windows of application. Application displays the stored data as shown in figure $4 \mathrm{a}$ and figure $4 \mathrm{~b}$ shows the feature of viewing the cumulative graph of heart rate and blood pressure values.

\section{EXPERIMENTAL SETUP AND RESULTS}

The device was tested in a rural area of Andhra Pradesh, India in association with MediCiti group of hospitals. The device was set up in a health kiosk of the village. The mobile network available in the village was used to communicate between village and the doctor. Figure 5a shows the interaction of doctor with health workers/technician. Figure $5 \mathrm{~b}$ shows a trained technician using the tablet.

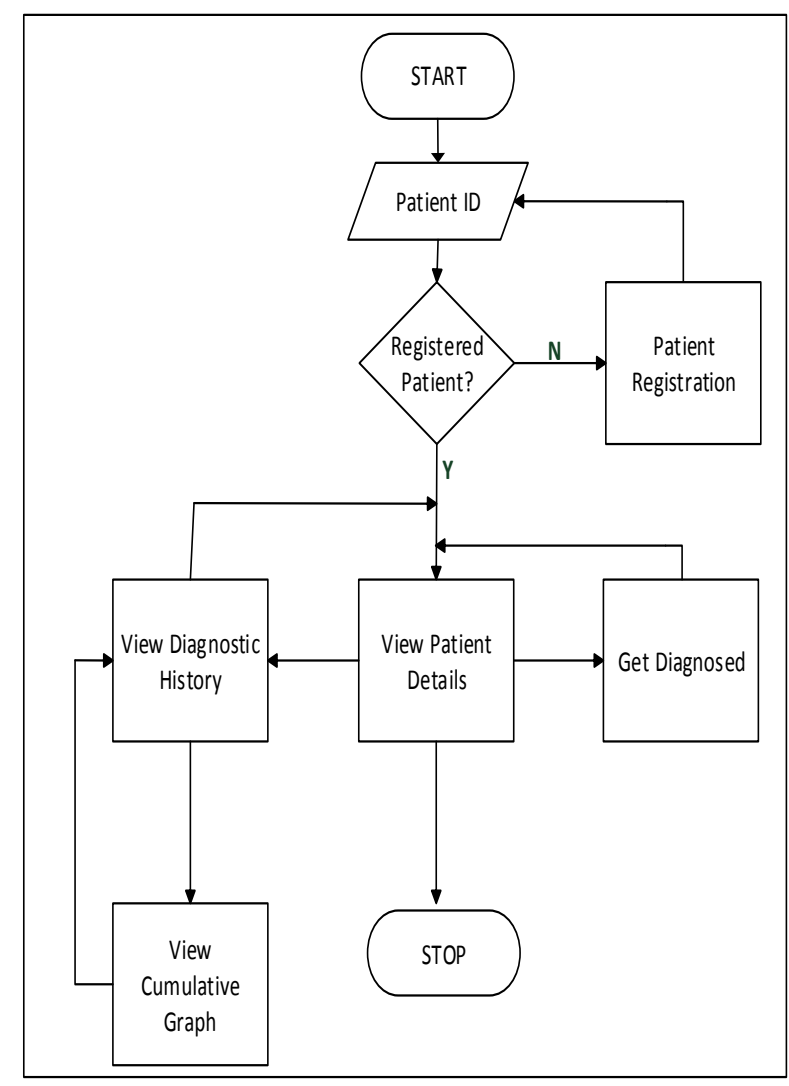

Figure 3. Flow chart of the android application

The trained technician registered the patient by collecting the details as mentioned in software part of the 


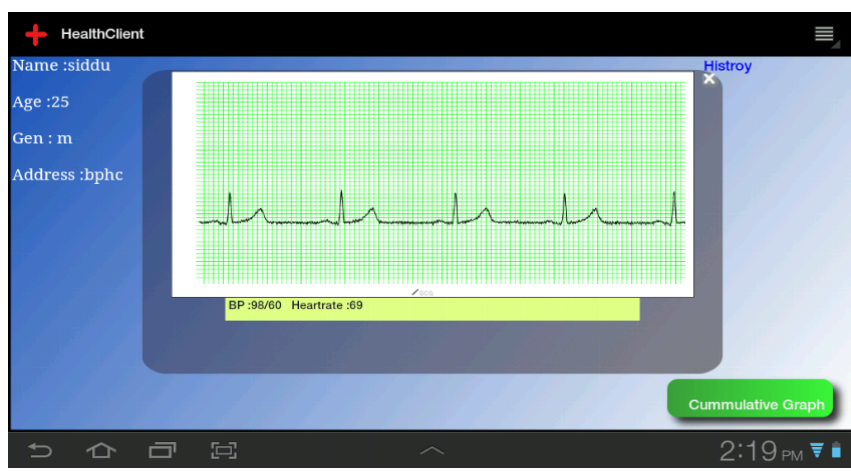

(a)

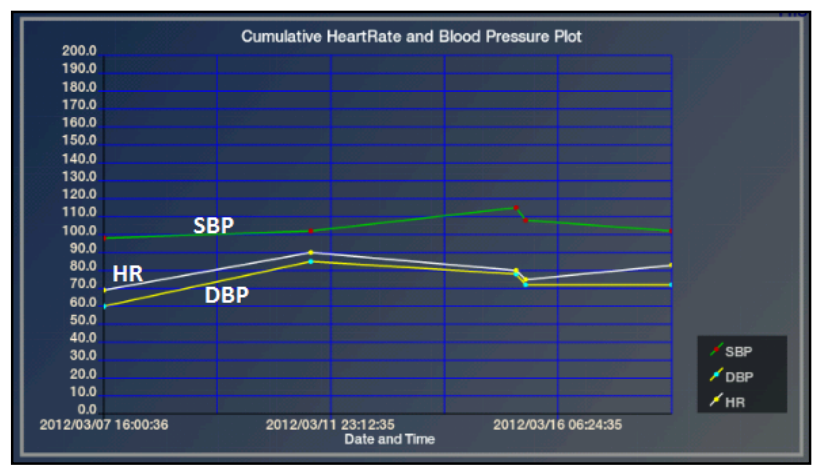

(b)

Figure 4. Pages of the software on android platform (a) Stored diagnostic data (b) cumulative graph of blood pressure and heart rate

implementation section. The personal details collected is stored on the cloud. Later the diagnostic data is collected as described in the previous section. The data collected is stored on the cloud. The details stored is retrieved by the doctor and also an audio/video call is established using the developed device.

The developed device has proved to support the rural healthcare system through remote diagnosis. The feature of

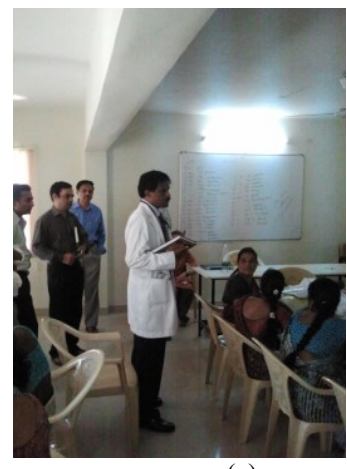

(a)

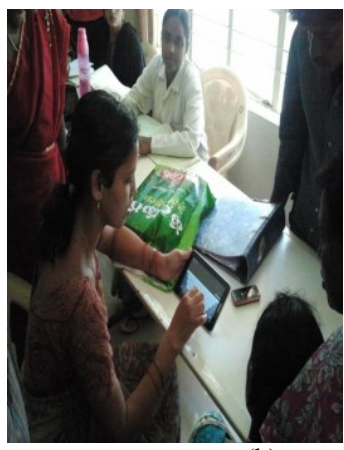

(b)
Figure 5. Health workers being trained (a) doctor speaking to health workers (b) a trained health worker using the tablet

audio/video call supports the doctors to provide a better rural healthcare in a cost effective way. The use of the technology has proved to provide a better healthcare to the rural population of developing countries

\section{ACKNOWLEDGEMENT}

Authors thank MediCiti group of hospitals, Hyderabad for providing support to test the platform and for their valuable inputs while developing the system.

\section{REFERENCES}

[1] "2011 Global medical trends", http://www.towerswatson.com/assets/pdf/3583/Towers-WatsonGlobal-Medical-Trends-Svy-Rpt.pdf

[2] "Using Mobility to Improve Patient Interactions," Sybase, http://m.sybase.com/detail?id=1093536

[3] "ehealthcare patient management without walls breaking", Oracle healthcare,

http://www.oracle.com/us/industries/healthcare/ehealthcare-patientmanagement-wp-173073.pdf

[4] "Vodafone mHealth", Vodafone, http://mhealth.vodafone.com/global/discover_mhealth/history_heritag e/index.jsp

[5] "Portable ECG recoder", Omron, http:/www.omronhealthcare.com/products/portable-ecg-monitor/

[6] "Electronic Stethoscope", 3M Littmann, http://solutions.3m.com/wps/portal/3M/en_WW/Littmann_3100_3200 /stethoscope/

[7] Hannu Sorvoja, Risto Myllylä, "Noninvasive blood pressure measurement methods," Molecular and Quantum Acoustics, vol. 27, pp. 239-264, 2006.

[8] C.C.Y. Poon, Y.T. Zhang, "Cuff-less and noninvasive measurements of arterial blood pressure by pulse transit time," Engineering in Medicine and Biology 27th Annual Conference, pp 5877-5880, 2005.

[9] Li Dingli, Pan Yi, Chen Hang, Ye Shuming, Yan Hong, "The establishment of a non-invasive continuous blood pressure measure system based on pulse transit time", unpublished.

[10] N.H. Mahmood, N.A. Zakaria, N.B. Sharifmuddin , S.N. Jalaludin, "Pulse wave transit time and its relationship with systolic blood pressure", International Conference on Mechanical and Electronics Engineering, vol. 2, pp. V2-183- V2-186, 2010.

[11] Soo-young Ye, Gi-Ryon Kim, Dong-Keun Jung, Seong-wan Baik, and Gye-rok Jeon, "Estimation of systolic and diastolic pressure using the pulse transit time," World Academy Of Science, Engineering And Technology, vol. 67, pp. 726-731, 2010.

[10] "USB Host," Android Dev Guide, http://developer.android.com/guide/index.html

[11] "IMSDroid," Doubango, http://code.google.com/p/imsdroid 